COMUNICACIÓN CIENTÍFICA IX CONGRESO NACIONAL DE LA SOCIEDAD CIENTÍFICA ESPAÑOLA DE ENFERMERÍA - SCELE. Mayo de 2018. UNIVERSIDAD DE ALICANTE.

\title{
METAS DE LA SEGURIDAD DEL PACIENTE APLICADA A BLOQUE QUIRÚRGICO
}

Ma José Utiel Peñaranda

F. Ruiz Jiménez

Hospital Universitario de Torrevieja. 


\section{PALABRAS CLAVE:}

patient security; medical error; process error; protocol; organization; safety.

\section{OBJETIVOS:}

General:

Fomentar la cultura de seguridad del paciente entre los profesionales sanitarios del bloque quirúrgico de los Hospitales universitarios de Vinalopó y Torrevieja, para cumplir las recomendaciones sobre seguridad del paciente propuestas por la Organización Mundial de la Salud (OMS).

Especificos:

Presentar el plan de acción para el cumplimiento de las medidas de seguridad del paciente dentro del bloque quirúrgico.

\section{MATERIAL Y MÉTODO:}

Mediante una búsqueda bibliográfica en las principales bases de datos (Pubmed, Cochrane y Scielo), se revisaron las principales metas de Seguridad del paciente y las especificas del bloque quirúrgico. Una vez analizadas se priorizaron para poder ser presentadas al equipo quirúrgico así como para implementarlas, siendo estas las siguientes:

- Transmisión de información mediante técnica SAER

- Hoja de relevo estandarizada.

- Glosario de abreviaturas

- Comprobación y manejo de la pulsera identificativa del paciente.

- Comunicación redundante

- Etiquetado de medicación, alto riesgo y "look alike"

- Registro de caducidad en viales multidosis

- Firma de lateralidad por cirujano.

- Check-list.

- Códigos de alertas en la historia clínica digitalizada.

- Fomento del programa de higiene de manos mediante cartelería y formación.

- Guías sobre el uso adecuado de antisépticos.

\section{RESULTADOS:}

Quedan plasmados de manera práctica y real todos los objetivos y metas de seguridad del paciente planteadas mostrando las diferentes herramientas implementadas por nuestro centro hospitalario.

Dar a conocer nuestra propia adaptación a dichas indicaciones en el bloque quirúrgico durante el proceso de acreditación Joint Comission International de nuestro centro de trabajo durante el 2017.

\section{CONCLUSIONES:}

La seguridad del paciente es un principio fundamental de la atención sanitaria. La mejora de la seguridad del paciente requiere un esfuerzo complejo por parte de toda la Organización, que abarca una amplia gama de acciones para conseguir la mejora del desempeño; la gestión de la seguridad y los riesgos ambientales, el control de las infecciones, el uso seguro de los medicamentos, y la seguridad de los equipos, de la práctica clínica y del entorno en el que se presta la atención sanitaria.

La enfermería debe trabajar siempre aplicando todas las medidas de seguridad disponibles para hacer efectiva la práctica basada en la evidencia. Debemos trabajar de acuerdo a standares de calidad, aplicando la evidencia científica a la práctica clínica e invitar a los profesionales de la enfermería a actualizarse a través de bibliografía disponible en portales (tales como Pubmed, Cochrane, Scielo y Joint Commission International, entre otros) con el fin de trabajar con el máximo nivel de seguridad, eficiencia y excelencia técnica que la investigación pone a nuestro alcance. 